\title{
Investigation of in vitro activity of colistin and tygecyclin against
}

\section{Stenotrophomonas maltophilia isolates}

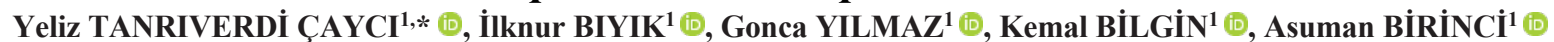

${ }^{1}$ Department of Medical Microbiology, Faculty of Medicine, Ondokuz Mayıs University, Samsun, Turkey

\begin{abstract}
\begin{tabular}{ccccc}
\hline Received: 02.04.2021 & $\bullet$ & Accepted/Published Online: 04.05.2021 & Final Version: 30.08 .2021 \\
\hline
\end{tabular}
Abstract

Stenotrophomonas maltophilia has emerged as an important opportunistic pathogen, causing infections whose management is often problematic due to its inherent resistance to many antibiotics. In this study, we aimed to investigate the antimicrobial susceptibility of colistin and tygecyclin as an alternative treatment options for S. maltophilia infections. A total of 122 S. maltophilia isolates were tested. Minimum inhibitory concentration (MIC) values of colistin and tygecycline were determined by broth microdilution method. Susceptibility of TMP/SMX and levofloxacin (LVX) were determined by disc diffusion method and MIC value of ceftazidime (CAZ) was determined by using E-test. Out of 122 S. maltophilia isolates, 5 (4\%) of them were resistant to TMP-SXM. MIC range was $0.125->512 \mu \mathrm{g} / \mathrm{ml}$ and $\mathrm{MIC}_{50} 64 \mu \mathrm{g} / \mathrm{ml}, \mathrm{MIC} 90512 \mu \mathrm{g} / \mathrm{ml}$ for colistin. MIC range for tygecyclin was detected as $0.5->8, \mathrm{MIC}_{50} 2 \mu \mathrm{g} / \mathrm{ml}$ and $\mathrm{MIC}_{90} 8 \mu \mathrm{g} / \mathrm{ml}$. Tygecyclin resistance was detected as $66.4 \%$ according to the EUCAST guideline and $13.1 \%$ according to the USA-FDA breakpoints. And colistin resistance was determined as $86.9 \%$ according to both guidelines.
\end{abstract}

Keywords: colistin, MIC, S. maltophilia, tygecyclin

\section{Introduction}

Stenotrophomonas maltophilia is an important nosocomial pathogen in certain patient populations, particularly in individuals who are immunosuppressed (1). S. maltophilia usually appears in immunocompromised and intensive care unit (ICU) patients, also frequently recovered from the respiratory tract of cystic fibrosis patients, and generally associated with infections of the respiratory tract, the organism is also a cause of bacteremia, endocarditis and urinary tract infections $(2,3)$. S. maltophilia infections are associated with high morbidity and mortality with the risk of mortality highest amongst patients receiving inappropriate initial antimicrobial therapy (4).

S. maltophilia is commonly resistant to several antimicrobial agents, including beta-lactams, due to heterogeneous production of b-lactamases (1). Reduced permeability and expression of efflux pumps could enhance this resistance phenotype (5, 6). Trimethoprim/sulfamethoxazole (TMP/SMX) is the main antimicrobial of choice for the treatment of $S$. maltophilia infections with ticarcillin/clavulanate, ceftazidime, minocycline, fluoroquinolones, tigecycline, and the polymyxins are described as alternative therapies (7). TMP/SMX resistance has been described and as high as $10 \%$ of isolates in Europe $(8,9)$. Tigecycline is the first glycylcycline antimicrobial and licensed for clinical use. Tigecycline binds to the $30 \mathrm{~S}$ ribosomal subunit and inhibits synthesis of protein. It has a wide range of activity against both Gram-positive and Gram-negative organisms (9).

Colistin, also known as polymyxin $\mathrm{E}$, is an old antibiotic and has in vitro activity against some multi-resistant Gramnegative bacteria, including $P$. aeruginosa, A. baumannii and Klebsiella pneumoniae. Beta-lactams, aminoglycosides, or quinolones are ineffective, colistin, remain drugs of last choice (10). Colistin has also been shown to possess in vitro activity against $S$. maltophilia strains $(83 \%-88 \%)$ of the tested isolates were susceptible to colistin in two recent studies) $(8,11,12)$. The aim of this study was to assess the antimicrobial resistance in $S$. maltophilia against colistin and tigecycline.

\section{Materials and methods}

A total of $122 \mathrm{~S}$. maltophilia isolates recovered from hospitalized patients in medical, surgical wards and in intensive care units were tested. Bacterial identification was made by using standard algorithms (microscopy, culture characteristics, oxidase reaction) followed by an automated system (Vitek MS, bioMeriux USA). Minimum inhibitory concentration (MIC) values of colistin and tigecycline were determined for all isolates based on the Clinical Laboratory Standards Institute (CLSI) (13) broth microdilution method. For tigecycline susceptibility fresh cation-adjusted MuellerHinton agar was used. Susceptibility of TMP/SMX and levofloxacin (LVX) were determined by disc diffusion method and MIC value of ceftazidime (CAZ) was determined by using E-test. 
Tigecycline breakpoints established by the USA-FDA for Enterobacteriaceae $\quad(\leq 2 \quad / \quad \geq 8 \quad \mu \mathrm{g} / \mathrm{ml}$ for susceptibility/resistance) and EUCAST for Enterobacteriaceae $\quad(\leq 1 \quad / \quad>2 \quad \mu \mathrm{g} / \mathrm{ml} \quad$ for susceptibility/resistance) as well as colistin breakpoints established by the CLSI for P. aeruginosa ( $\leq 2 / \geq 8 \mu \mathrm{g} / \mathrm{ml}$ for susceptibility/resistance), and the EUCAST for P. aeruginosa $(\leq 4 />4 \mu \mathrm{g} / \mathrm{ml}$ for susceptibility/resistance), were applied for comparison purposes (14-16). MIC50 and MIC90 values were determined for each antimicrobial. TMP/SMX, LVX and CAZ susceptibilities were interpreted according to the CLSI criteria established for S. maltophilia (14).

\section{Results}

Clinical sites of infection for S. maltophilia were primarily bloodstream (35.3\%) and respiratory tract (33.6\%) (Table 1).

Table 1. Distribution of clinical specimen

\begin{tabular}{|c|c|}
\hline Specimen & $\mathbf{n}(\%)$ \\
\hline Respiratory tract & $41(33.6)$ \\
Bloodstream & $43(35.3)$ \\
Urine & $19(15.6)$ \\
Wound & $10(8.2)$ \\
Catheter tip & $5(4.1)$ \\
Sterile body fluid & $2(1.6)$ \\
Conjonktiva & $2(1.6)$ \\
\hline
\end{tabular}

Out of $122 \mathrm{~S}$. maltophilia isolates, $5(4 \%)$ of them were resistant to TMP-SXM. LVX and CAZ resistance were determined as $6.5 \%$ and $56.5 \%$, respectively.

MIC range was $0.125->512 \mu \mathrm{g} / \mathrm{ml}$ and $\mathrm{MIC}_{50} 64 \mu \mathrm{g} / \mathrm{ml}$, $\mathrm{MIC}_{90} 512 \mu \mathrm{g} / \mathrm{ml}$ for colistin. For tigecycline, MIC range was detected as $0.5->8, \mathrm{MIC}_{50} 2 \mu \mathrm{g} / \mathrm{ml}$ and $\mathrm{MIC}_{90} 8 \mu \mathrm{g} / \mathrm{ml}$. Tigecycline resistance was detected as $66.4 \%$ according to the EUCAST guideline and $13.1 \%$ according to the USA-FDA breakpoints. And colistin resistance was determined as $86.9 \%$ according to both guidelines (Table 2).

Susceptibility rates according to the clinical sites were specified in Table 3. Tigecycline susceptibility was determined highest in conjunctiva and sterile body fluids as $50 \%$, however the specimen number is very low $(\mathrm{n}=2)$ for these clinical sites. For bloodstream and respiratory tract specimens tigecycline susceptibility was determined as $39.5 \%-88.4 \%$ and $12.2 \%-78.0 \%$, according to the EUCAST and USA-FDA breakpoints, respectively. The highest colistin susceptibility were determined for bloodstream isolates as $21 \%$. And for catheter tips, conjunctiva and sterile body fluids tigecycline seems more effective than colistin in vitro.

Table 2. Resistance rates for tigecycline and colistin according to the EUCAST and CLSI/USA-FDA criteria

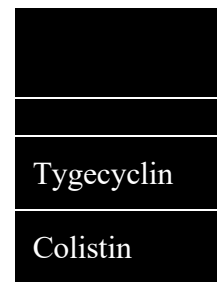

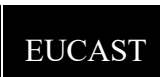

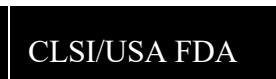

$\mathrm{MIC}_{50}$ $\mathrm{MIC}_{90}$
Table 3. Distribution of tigecycline and colistin susceptibilities according to the clinical specimens

\begin{tabular}{|l|l|l|} 
& Tygecyclin & Colistin (S) \\
& EUCAST(S) & EUCAST \\
& CLSI(S) & \\
& $39.5 \%$ & $21 \%$ \\
\multirow{2}{*}{ Blood stream (n:43) } & $88.4 \%$ & $21 \%$ \\
\hline \multirow{2}{*}{ Respiratory tract (n:41) } & $29.3 \%$ & $12.2 \%$ \\
\hline \multirow{2}{*}{ Urine (n:19) } & $78.0 \%$ & $12.2 \%$ \\
\hline \multirow{2}{*}{ Wound (n:10) } & $36.8 \%$ & $5.3 \%$ \\
& $79.0 \%$ & $5.3 \%$ \\
\hline \multirow{2}{*}{ Catheter tip (n:5) } & $30.0 \%$ & $10.0 \%$ \\
& $60.0 \%$ & $10.0 \%$ \\
\hline \multirow{2}{*}{ Sterile body fluid (n:2) } & $0 \%$ & $0 \%$ \\
& $60.0 \%$ & $0 \%$ \\
\multirow{2}{*}{ Conjunctiva (n:2) } & $50 \%$ & $0 \%$ \\
& $100 \%$ & $0 \%$ \\
\end{tabular}

\section{Discussion}

In this study, in vitro effectiveness of tigecycline and colistin was investigated as an alternative for treatment of $S$. maltophilia isolates that were isolated from clinical samples in our hospital.

S. maltophilia is accepted as a pathogen with gradually increasing importance recently. The reason for this may be increasing number of immune-compromised patients, prolonged hospital stays and increasing use of wide spectrum antibiotics like carbapenems (17). S. maltophilia was detected to be the most common non-fermentative bacillus following P. aeruginosa and Acinetobacter spp. between 1997-2001 and isolation ratio was found as $8 \%$ in clinical samples (18).

S. maltophilia may lead to respiratory tract, bloodstream, urinary tract and wound infections. In the studies, $S$. maltophilia was shown to be isolated from different sample types. While vast majority of the isolates were isolated from blood samples in this study, respiratory tract samples, urinary tract samples were the most common in some other studies (19-22).

TMP-SXM has been considered as the first therapeutic option against $S$. maltophilia infections, but this is primarily based on in vitro susceptibility data (7). However, increasing resistance to trimethoprim/sulfamethoxazole has been reported $(23,24)$, mostly related to the horizontal spread of mobile genetic elements which are carrying resistance genes $(25,26)$.

The fluoroquinolones are one of the other main alternative treatment options for the S. maltophilia infections (7). According to reports, primarily ciprofloxacin, levofloxacin and, particularly, moxifloxacin can have more potent in vitro activity $(15,27,29)$. Also, it is reported that resistance to the fluoroquinolones can arise during therapy. In our study, the in vitro resistance to levofloxacin was $6.5 \%$ (30-32). Among the beta lactams, ceftazidime is the agent that can be considered as potential therapeutic options against $S$. maltophilia 
infections (23). In our study, the susceptibility to this agent was rather low as $43.5 \%$, in agreement with other relevant studies (33).

New treatment options are required due to limited number of antimicrobial agents and resistance development against the agents used for treatment of $S$. maltophilia. Colistin and tigecycline are among these new options. In a study investigating colistin susceptibility in S. maltophilia isolates, colistin resistance was found to elevate to $60 \%$ in 2010 while it was $8 \%$ in 1996. Authors reported that this was associated with increasing use of colistin (34). Colistin resistance was found between $24-100 \%$ in a few studies which was conducted with small number of $S$. maltophilia isolates $(8$, 35-37). Samonis et al. (19) found colistin susceptibility as 91.2\%. The clinical breakpoints determined for P. aeruginosa by CLSI was used in these studies. Susceptibility method variabilities were reported as the reason for differences in the resistance rates (36). Geographic region and patient population were also reported to be able to be effective on colistin resistance profile $(33,37,38)$.

Insa et al. (39) investigated the effectiveness of tigecycline in $120 \mathrm{~S}$. maltophilia isolates and found susceptibility of isolates as $98 \%$ when they accepted breakpoint value as $\geq 2 \mu \mathrm{g} / \mathrm{ml}$. Farrel et al. (33) found tigecycline susceptibility as 95\% (USA-FDA criteria were used as limit value for tigecycline) in their study investigating tigecycline susceptibility in S. maltophilia isolates isolated from different regions of the world. Tigecyclin was found as the most effective agent following TMP-SXM also in this study. Colistin susceptibility was found as $64.6 \%$ in the same study (33). Absence of a clinical breakpoint value for $S$. maltophilia in categorical assessment of tigecycline susceptibility and use of different clinical breakpoint influence resistance rates (1416). When tigecycline susceptibility was evaluated in this study, while resistance rate is $13.1 \%$ according to USA-FDA criteria, this value elevated to $66.4 \%$ according to EUCAST criteria.

Absence of a specified clinical breakpoints for assessment of colistin and tigecycline susceptibility for S. maltophilia isolates seems to be one of the reasons for detecting different susceptibility rates. Determination of clinical breakpoints against these agents with future studies conducted with larger series is suggested to be useful for treatment of S. maltophilia infections which is gradually increasing.

\section{References}

1. Denton M, Kerr KG. Microbiological and Clinical Aspects of Infection Associated with Stenotrophomonas maltophilia. Clın Microb Rev. 1998;11(1): 57-80.

2. Zhang L, Li XZ, Poole K. Multiple antibiotic resistance in Stenotrophomonas maltophilia: involvement of a multidrug efflux system. Antimicrob Agents Chemother. 2000; 44:287-93.

3. Valdezate S, Vindel A, Loza E, Baquero F, Canto'n R. Antimicrobial susceptibilities of unique Stenotrophomonas maltophilia clinical strains. Antimicrob Agents Chemother. 2001;45: 1581-4.

4. Falagas ME, Kastoris AC, Vouloumanou EK, Rafailidis PI, Kapaskelis AM, Dimopoulos G. Attributable mortality of Stenotrophomonas maltophilia infections: a systematic review of the literature. Future Microbiology. 2009; 4:1103-9.

5. Alonso A, Martı'nez JL. Multiple antibiotic resistance in Stenotrophomonas maltophilia. Antimicrob Agents Chemother. 1997; 41:1140-2.

6. Yamazaki E, Ishii J, Sato K, Nakae T. The barrier function of the outer membrane of Pseudomonas maltophilia in the diffusion of saccharides and beta-lactam antibiotics. FEMS Microbiol Lett. 1989; 51:85-8.

7. Nicodemo AC, Paez JI. Antimicrobial therapy for Stenotrophomonas maltophilia infections. Eur J Clin Microbiol Infect Dis. 2007; 26: 229- 37.

8. Gales AC, Jones RN, Forward KR, Linares J, Sader HS, Verhoef J. Emerging importance of multidrug-resistant Acinetobacter species and Stenotrophomonas maltophilia as pathogens in seriously ill patients: geographic patterns, epidemiological features, and trends in the SENTRY Antimicrobial Surveillance Program (1997-1999). Clin Infect Dis. 2001; 32 Suppl 2:104-13.

9. Petersen PJ, Jacobus NV, Weiss WJ, Sum PE, Testa RT. In vitro and in vivo antibacterial activities of a novel glycylcycline, the 9t10 butylglycylamido derivative of minocycline (GAR-936). 174 Antimicrob Agents Chemother. 1999; 43:738-44.

10. Li J, Nationa RL, Milneb RW, Turnidgec JD, Coulthard K. Evaluation of colistin as an agent against multi-resistant Gramnegative bacteria. International Journal of Antimicrobial Agents. 2005; 25 : $11-25$.

11. Gales AC, Reis AO, Jones RN. Contemporary assessment of antimicrobial susceptibility testing methods for polymyxin B and colistin: review of available interpretative criteria and quality control guidelines. J Clin Microbiol. 2001; 39: 183-90.

12. Hogardt M, Schmoldt S, Gotzfried M, Adler K, Heesemann J. Pitfalls of polymyxin antimicrobial susceptibility testing of Pseudomonas aeruginosa isolated from cystic fibrosis patients. J Antimicrob Chemother. 2004; 54:1057-61.

13. Clinical and Laboratory Standarts Institute. Methods for Dilution Antimicrobial Susceptibility Tests for Bacteria That Grow Aerobically; Approved Standard-Ninth Edition, M07-A9. 2012; 32(2).

14. Clinical and Laboratory Standards Institute: Performance Standards for Antimicrobial Susceptibility Testing, Twentieth Informational Supplement, M100-S24, CLSI, 2014, Wayne, PA.

15. EUCAST.http://www.eucast.org/fileadmin/src/media/PDFs/EUC AST_files/Breakpoint_tables/v_6.0_Breakpoint_table.pdf (Accessed 31.03.2021).

16. Tygacil. Wyeth Pharmaceuticals, Philadelphia, PA. 2009.

17. Dizbay M, Tunçcan ÖG, Maral I, Aktaş F, Şenol E. Five year surveillance of nosocomial Stenotrophomonas maltophilia infections in Gazi University Hospital. Türkiye Klinikleri J Med Sci. 2009; 29: 1406-11.

18. Jones RN, Sader HS, Beach ML. Contemporary in vitro spectrum of activity summary for antimicrobial agents tested against 18569 strains non-fermentative gram negative bacilli isolated in the SENTRY Antimicrobial Surveillance Program (1997-2001). Int J Antimicrob Agents. 2003; 22: 551-6.

19. Samonis G, Karageorgopoulos DE, Maraki S, Levis $P$, Dimopoulou D, Spernovasilis NA et al. Stenotrophomonas maltophilia Infections in a General Hospital: Patient 
Characteristics, Antimicrobial Susceptibility, and Treatment Outcome. PLoS ONE. 2012 ;7(5): e37375.

20. Öngüt G, Özcan A, Kandişer A, Öğünç D, Çolak D, Gültekin M. Çeşitli klinik örneklerden izole edilen Stenotrophomonas maltophilia suşlarının antimikrobiyal duyarlılıklarının E Test ile araştırılması (in Turkish). İnfeks Derg. 2005; 19: 425-8.

21. Dülger D, Berktaş $M$, Bozkurt $H$, Güdücüoğlu $H$, Mısırlıgil A. Nozokomiyal Stenotrophomonas maltophilia suşlarının izolasyonu ve antibiyotiklere duyarlılığı (in Turkish). Van Tıp Derg. 2006; 13:49-52.

22. Zer Y, Karaoğlan İ, Çevik S, Erdem M. Stenotrophomonas maltophilia suşlarının antibiyotik duyarlılıklarının irdelenmesi (in Turkish). Klimik Derg. 2009; 22: 21-4.

23. Penzak SR, Abate BJ. Stenotrophomonas (Xanthomonas) maltophilia: a multidrug-resistant nosocomial pathogen. Pharmacotherapy. 1997; 17: 293-301.

24. Crossman LC, Gould VC, Dow JM, Vernikos GS, Okazaki $\mathrm{A}$, Sebaihia $\mathrm{M}$ et al. The complete genome, comparative and functional analysis of Stenotrophomonas maltophilia reveals an organism heavily shielded by drug resistance determinants. Genome Biol. 2008; 9: R74.

25. Toleman MA, Bennett PM, Bennett DM, Jones RN, Walsh TR. Global emergence of trimethoprim/sulfamethoxazole resistance in Stenotrophomonas maltophilia mediated by acquisition of sul genes. Emerg Infect Dis. 2007; 13: 559-65.

26. Hu LF, Chang X, Ye Y, Wang ZX, Shao YB, Shi W, et al. Stenotrophomonas maltophilia resistance to trimethoprim/sulfamethoxazole mediated by acquisition of sul and dfrA genes in a plasmid-mediated class 1 integron. Int $\mathrm{J}$ Antimicrob Agents. 2011; 37: 230-4.

27. Looney WJ, Narita M, Muhlemann K. Stenotrophomonas maltophilia: an emerging opportunist human pathogen. Lancet Infect Dis. 2009; 9: 312-23.

28. Falagas ME, Valkimadi PE, Huang YT, Matthaiou DK, Hsueh PR. Therapeutic options for Stenotrophomonas maltophilia infections beyond cotrimoxazole: a systematic review. J Antimicrob Chemother. 2008; 62: 889-94.

29. Vartivarian S, Anaissie E, Bodey G, Sprigg H, Rolston K. A changing pattern of susceptibility of Xanthomonas maltophilia to antimicrobial agents: implications for therapy. Antimicrob Agents Chemother. 1994; 38: 624-7.

30. Garrison MW, Anderson DE, Campbell DM, Carroll KC,
Malone CL, Anderson JD et al. Stenotrophomonas maltophilia: emergence of multidrug-resistant strains during therapy and in an in vitro pharmacodynamic chamber model. Antimicrob Agents Chemother. 1996; 40: 2859-64.

31. Valdezate S, Vindel A, Saez-Nieto JA, Baquero F, Canton R. Preservation of topoisomerase genetic sequences during in vivo and in vitro development of high-level resistance to ciprofloxacin in isogenic Stenotrophomonas maltophilia strains. J Antimicrob Chemother. 2005; 56: 220-3.

32. Lecso-Bornet M, Pierre J, Sarkis-Karam D, Lubera S, BergogneBerezin E. Susceptibility of Xanthomonas maltophilia to six quinolones and study of outer membrane proteins in resistant mutants selected in vitro. Antimicrob Agents Chemother. 1992; 36: 669-71.

33. Farrell DJ, Sader HS, Jones RN. Antimicrobial susceptibilities of a worldwide collection of Stenotrophomonas maltophilia isolates tested against tigecycline and agents commonly used for $\mathrm{S}$. maltophilia infections. Antimicrob Agents Chemother. 2010; 54: 2735-7.

34. Rodrı'guez CH, Nastro M, Lopez Calvo J, Farin ME, Dabos L, Famiglietti A. In vitro activity of colistin against Stenotrophomonas maltophilia. J Glob Antimicrob Resist. 2014: 316-7.

35. Moskowitz SM, Garber E, Chen Y, Clock SA, Tabibi S, Miller AK et al. Colistin susceptibility testing: evaluation of reliability for cystic fibrosis isolates of Pseudomonas aeruginosa and Stenotrophomonas maltophilia. J Antimicrob Chemother. 2010; 65: 1416-23.

36. Nicodemo AC, Araujo MR, Ruiz AS Gales AC. In vitro susceptibility of Stenotrophomonas maltophilia isolates: comparison of disc diffusion, Etest and agar dilution methods. J Antimicrob Chemother. 2004; 53: 604-8.

37. Tan TY, Ng SY. Comparison of Etest, Vitek and agar dilution for susceptibility testing of colistin. Clin Microbiol Infect. 2007; 13: $541-4$.

38. San Gabriel P, Zhou J, Tabibi S, Chen Y, Trauzzi M, Saiman L. Antimicrobial susceptibility and synergy studies of Stenotrophomonas maltophilia isolates from patients with cystic fibrosis. Antimicrob Agents Chemother. 2004; 48: 168-71.

39. Insa R, E. Cercenado E, Goyanes MJ, Morente A, Bouza E. In vitro activity of tigecycline against clinical isolates of Acinetobacter baumannii and Stenotrophomonas maltophilia. J Antimicrob Chemother. 2007;59 (3): 583-5. 